\title{
Comparative genomics and community curation further improve gene annotations in the nematode Pristionchus pacificus
}

\author{
Marina Athanasouli, Hanh Witte, Christian Weiler, Tobias Loschko, Gabi Eberhardt, Ralf J. Sommer and \\ Christian Rödelsperger *i)
}

\begin{abstract}
Background: Nematode model organisms such as Caenorhabditis elegans and Pristionchus pacificus are powerful systems for studying the evolution of gene function at a mechanistic level. However, the identification of $P$. pacificus orthologs of candidate genes known from C. elegans is complicated by the discrepancy in the quality of gene annotations, a common problem in nematode and invertebrate genomics.

Results: Here, we combine comparative genomic screens for suspicious gene models with community-based curation to further improve the quality of gene annotations in P. pacificus. We extend previous curations of one-toone orthologs to larger gene families and also orphan genes. Cross-species comparisons of protein lengths, screens for atypical domain combinations and species-specific orphan genes resulted in 4311 candidate genes that were subject to community-based curation. Corrections for 2946 gene models were implemented in a new version of the $P$. pacificus gene annotations. The new set of gene annotations contains 28,896 genes and has a single copy ortholog completeness level of $97.6 \%$.

Conclusions: Our work demonstrates the effectiveness of comparative genomic screens to identify suspicious gene models and the scalability of community-based approaches to improve the quality of thousands of gene models. Similar community-based approaches can help to improve the quality of gene annotations in other invertebrate species, including parasitic nematodes.
\end{abstract}

Keywords: Genome, Evolution, Caenorhabditis elegans, Parasitic nematodes, Orphan genes

\section{Background}

The nematode Pristionchus pacificus was initially introduced as a satellite model organism for comparing developmental processes to Caenorhabditis elegans $[1,2]$. More recently, it has emerged as an independent model organism for studying the genetics of phenotypic plasticity $[3-5]$ and behavior [6-8], interactions between host and microbes [9-11], and genome evolution [12-14]. Central to all these studies was the genome sequence of $P$.

\footnotetext{
* Correspondence: christian.roedelsperger@tuebingen.mpg.de Department for Integrative Evolutionary Biology, Max Planck Institute for Developmental Biology, Max-Planck-Ring 9, 72076 Tübingen, Germany
}

pacificus, which has undergone continuous improvements over time [15-17]. However, until recently, its gene annotations were almost exclusively based on automated pipelines that combined gene predictions and evidence-based annotations [18-20]. As a consequence, the gene annotations of $P$. pacificus did not match the quality of the highly curated C. elegans genome. This made it difficult for researchers from the $C$. elegans field to adapt $P$. pacificus for comparative studies, even though the availability of genetic toolkits including transgenic reporter lines and gene knockouts makes $P$. pacificus ideally suited for comparative studies of gene function $[8,21,22]$. Therefore, we

(c) The Author(s). 2020 Open Access This article is licensed under a Creative Commons Attribution 4.0 International License, which permits use, sharing, adaptation, distribution and reproduction in any medium or format, as long as you give appropriate credit to the original author(s) and the source, provide a link to the Creative Commons licence, and indicate if changes were made. The images or other third party material in this article are included in the article's Creative Commons licence, unless indicated otherwise in a credit line to the material. If material is not included in the article's Creative Commons licence and your intended use is not permitted by statutory regulation or exceeds the permitted use, you will need to obtain permission directly from the copyright holder. To view a copy of this licence, visit http://creativecommons.org/licenses/by/4.0/. The Creative Commons Public Domain Dedication waiver (http://creativecommons.org/publicdomain/zero/1.0/) applies to the data made available in this article, unless otherwise stated in a credit line to the data. 
have recently started to combine comparative genomic screens for suspicious gene models with communitybased manual curation to improve the quality of the gene annotations in P. pacificus [23]. This pilot study screened for missing one-to-one orthologs of C. elegans genes in $P$. pacificus. Community-based curation of these candidate gene loci resulted in a substantial improvement of the $P$. pacificus gene annotations (version: El Paco annotation V2). Precisely, when assessed by benchmarking of universally conserved single copy orthologs (BUSCO) [24], the completeness level increased from 86 to $97 \%$. Most missing orthologs were due to fused gene models some of which had long untranslated regions (UTRs) that actually contained complete genes. These errors could be corrected by manual inspection of the suspicious gene loci under the consideration of two recent transcriptome assemblies that were generated from strand-specific RNA-seq [25, 26] and Iso-seq data [27].

Here, we employ comparative genomic approaches to screen for further errors in other gene classes including large gene families that have undergone lineage-specific duplications [28] and species-specific orphan genes (SSOGs) [29] that were not the focus of our previous study [23]. Candidate loci are then curated by community-based manual inspection and eventually, corrections were proposed mainly based on available transcriptome assemblies. Overall, we investigated 4311 suspicious gene models and implemented 2946 corrections. This resulted in a further improved set of gene annotations for P. pacificus. Similar community-based curation approaches can help approving gene annotations in other nematode genomes including those of animal and plant parasites [23].

\section{Results}

Protein length comparison of orthologs identify hundreds of suspicious gene models

In our previous study, we focused on the identification of missing one-to-one orthologous genes in the P. pacificus genome and the identification of artificial fusions between two adjacent $P$. pacificus genes both of which have one-to-one orthologous genes [23]. Here, we aim to further improve the quality of one-to-one orthologous genes by finding and curating $P$. pacificus genes that are either unusually large or small with regard to their $C$. elegans counterpart. We performed a comparison of protein length of 8348 one-to-one orthologs between $C$. elegans and P. pacificus (Fig. 1a-c). Protein lengths between one-to-one orthologs are well correlated (Pearson's $r=0.83$, Fig. 1a). However, there are slight differences in the length distributions (Fig. 1b,c) and using an arbitrary cutoff of a two-fold difference in protein length, we defined $532 P$. pacificus genes as candidates for manual inspection. For example, in the case of the $P$. pacificus gene PPA00494 (ortholog of C. elegans lev-8), its predicted protein sequence encompasses 1094 amino acids, which is more than twice as long as C. elegans LEV-8 (531 amino acids) (Fig. 1d). Also, BLASTP analysis against the C. elegans proteins (version WS277) shows that the N-terminal part of PPA00494 is homologous to another C. elegans protein, Y73B6BL.37 (Fig. 1d), suggesting that it could represent an artificial gene fusion. Subsequent inspection in the genome browser showed two transcripts that were assembled from strandspecific RNA-seq data [26], which span the PPA00494 locus (Fig. 1e). This strongly supports that PPA00494 should be split by replacing it with the two assembled transcripts. After community-based curation, 309 (57\%) corrections were proposed. The remaining cases were judged as either inconclusive (due to the lack of transcriptomic support) or correct. These results demonstrate that protein length comparisons between one-to-one orthologs are an effective way to identify suspicious gene models and to further improve the quality of one-to-one orthologs.

\section{Analysis of protein domains identifies further artificial gene fusions}

Our previous study showed that the combination of incorrectly predicted gene boundaries and overlapping UTRs between neighboring genes in regions with high gene density most likely caused artificial gene fusions. In order to screen for further cases of artificial gene fusions, we applied a comparative genomic approach to identify proteins with atypical domain combinations that do not exist in other nematodes such as C. elegans, and more distantly related Bursaphelenchelus xylophilus [30], and Strongyloides ratti [31]. This yielded 1589 P. pacificus candidates (Table 1) for further inspection. Note, that such atypical domain combinations are not necessarily artifacts. For example, the same screen in the highly curated C. elegans genome, identified 932 genes with atypical domain combinations. Manual inspection of these gene models and available transcriptome assemblies in the WormBase genome browser (WS177) combined with BLASTP analysis against $C$. briggsae revealed three candidates for putatively incorrect annotation in C. elegans, which deserve closer inspection (Additional file 1, Figure S1). After community curation of the $P$. pacificus candidates, corrections were proposed for 695 (44\%) candidates. Next, we defined 1388 unusually small or long members of 25 highly abundant gene families as further candidates for manual inspection (Fig. 2a). After community curation, corrections were proposed for 420 (32\%) of these candidates. The three described screens partially identify the same candidates (Fig. $2 \mathrm{~b}$ ), yet the presence of hundreds of candidate genes that are specific to each method indicates how complementary these different approaches are.

\section{Gene prediction artifacts are a likely source of SSOGs}

A previous analysis of $P$. pacificus orphan genes revealed that the majority of SSOGs had no transcriptomic support 


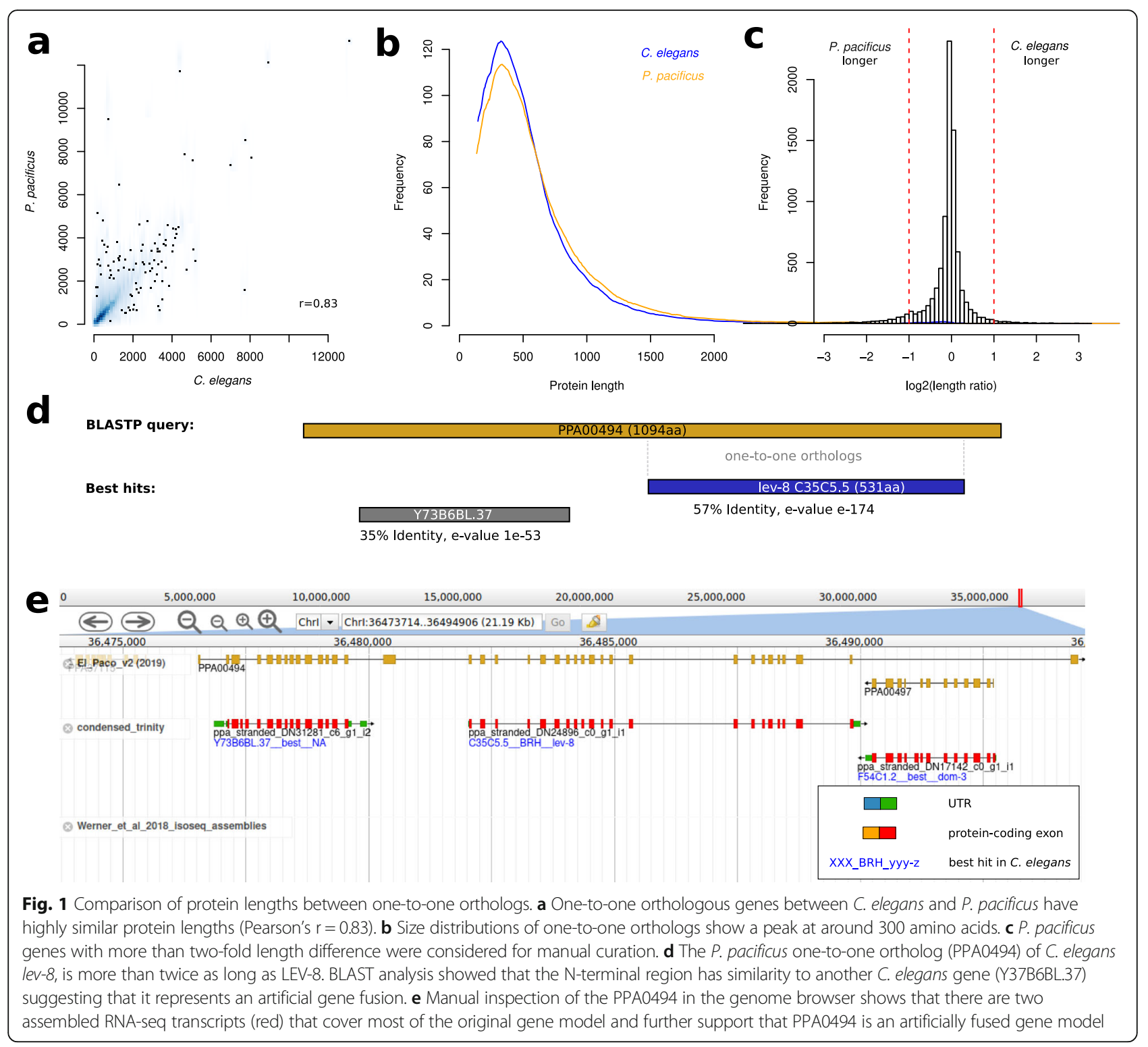

[14]. Based on the reanalysis of the current gene annotations with available phylogenomic and phylotranscriptomic data [26, 32], we identified 1988 (7\%) P. pacificus SSOGs of which 314 were classified as having transcriptomic support. Manual inspection of the remaining SSOGs classified 678 (41\%) of candidates as not having any transcriptional support (Fig. 2c), even when considering additional transcriptomic data sets such as iso-seq or dauer-specific transcriptomes [27, 33]. Further 196 (12\%) of SSOG candidates showed some transcriptional activity, but this expression data was mostly not sufficient to support their gene structure. Strikingly, we found 704 (42\%) and 46 (3\%) SSOGs, which overlapped existing gene models on the antisense strand of protein-coding exons and UTRs, respectively (Fig. 2c and 3a,b). However, visual inspection of transcriptomic data only supported the sense gene as opposed to the antisense SSOG. As there is neither protein homology nor transcriptional data supporting these antisense SSOGs, we would tend to argue that these spurious antisense gene models most likely derive from the contribution of gene prediction softwares SNAP and AUGUSTUS during the process of the original gene annotation [17, 19, 20]. Thus, manual curation removed 1515 of the unsupported SSOGs, mainly from the "no support", "Antisense prediction", and "UTR" categories (Fig. 2c), as their lack of transcriptional evidence makes it difficult to conclusively study the process of novel gene formation $[14,29]$.

\section{New P. pacificus gene annotations show increased} homogeneity and better reflect existing RNA-seq data In total, we visually inspected 4311 suspicious gene models and proposed corrections for 2946 (68\%). The 
Table 1 Comparative assessment of different $P$. pacificus gene annotations

\begin{tabular}{lll}
\hline Category & \multicolumn{2}{l}{$\begin{array}{l}\text { P. pacificus El Paco } \\
\text { gene annotations }\end{array}$} \\
\cline { 2 - 3 } & V2 & V3 \\
\hline Number of genes & 28,036 & 28,896 \\
Protein-coding sequence (Mb) & 35.3 & 35.3 \\
BUSCO Completeness (\%) & 97.1 & 97.6 \\
BUSCO Duplicated (\%) & 1.7 & 1.8 \\
BUSCO Fragmented (\%) & 2.0 & 2.0 \\
BUSCO Missing (\%) & 0.9 & 0.4 \\
Number of 1-1 orthologs (BRHs) & 8348 & 8607 \\
Number of 1-1 orthologs with & 532 & 265 \\
variable protein length (\%) & & 1137 \\
Number of proteins with atypical & 1589 & 1201 \\
domain combinations & & \\
Number of protein family length & 1388 & \\
outlier & &
\end{tabular}

The table shows an overview about general characteristics of different $P$. pacificus gene annotations

most common errors were artificial fusions and unsupported SSOGs that are putative annotations artifacts. This led to the correction of 1367 gene models by replacing them with one or more alternative gene models and to the removal of 1579 gene models. We implemented all proposed corrections into a new $P$. pacificus gene annotation (version: El Paco gene annotation V3), which comprises 28,896 gene models and spans $35.2 \mathrm{Mb}$ of protein-coding sequence with a BUSCO completeness level of $97.6 \%$ (Table 1). As expected, the numbers of oneto-one orthologs with length differences, the number of genes with atypical domain combinations, and the number of gene family outliers went down by $10-50 \%$. To additionally test if the new set of gene annotations better captures RNA-seq data sets, we reanalyzed 15 RNA-seq data sets from four different studies [9, 13, 34, 35] and quantified the percentage of reads that could be assigned to features of the gene annotations. The new set of gene annotations consistently captures $2 \%$ more of the RNAseq alignments (Table 2). Despite the fact that the number of genes was increased by $\sim 3 \%$, the total amount of annotated protein-coding sequence remained almost unaltered (Table 1). Thus, the new set of gene annotations better reflects RNA-seq data.

\section{Discussion}

In the early genomic era, gene annotation was heavily dependent on automated gene finding algorithms that tried to recognize gene structures based on statistical sequence properties of exons, introns, and splicing sites $[19,20]$. This was highly suited when functional data, e.g. expressed sequence tags and cDNAs, were scarce and the only way to annotate a complete genome was to extract informative sequence features from a limited test set and extrapolate them to the whole genome. However, with the dramatic improvement of sequencing protocols and technologies, it became feasible to generate evidence-based gene annotations from transcriptome and homology data $[18,36]$. Under the consideration that related genomes at an optimal evolutionary distance to a focal organism and transcriptomic evidence for all genes are rarely available, this still justifies the usage of gene prediction tools. In the case of the $P$. pacificus, previous versions of gene annotations that were completely based on the results of gene prediction tools were suited to perform evolutionary genomic analysis and genetic screens $[37,38]$. Subsequently, we employed the widely used MAKER2 pipeline to generate a more comprehensive gene annotation by integration of large-scale transcriptomic and protein homology data as well as gene predictions $[17,36]$. Comparative analysis of genome quality for 22 nematode species revealed that already these gene annotations (version: El Paco annotation V1) were of relatively high quality (86\% BUSCO completeness) [23]. Nevertheless, the question of how good gene annotations need to be will depend on what researchers want to do with them. Reverse genetic studies in nematodes with well established genetic toolkits are extremely powerful systems for comparative studies of gene function $[8,22]$ and the evolution of the nervous system and associated behaviors $[39,40]$. Yet, the identification of $P$. pacificus orthologs for candidate $C$. elegans genes with known function is complicated by the widespread abundance of lineagespecific duplications [28, 33], but also by the difference in the quality of gene annotations. Facilitating the easy adaptation of $P$. pacificus as a comparative model system for $C$. elegans researchers, who are used to working with one of the best and well-characterized genomes, is one of our main motivations for this study. The chromosome-scale assembly of $P$. pacificus has already been a major step to minimize the disparity between the genomic resources of both species [17]. Lifting up the quality of gene annotations to a comparable level will thus further increase the attractiveness of the $P$. pacificus system for evolutionary studies.

Another motivation for continuous efforts in improving the quality of gene annotations is our focus on the origin and evolution of orphan genes in $P$. pacificus [41-43]. Initially, around one-third of the P. pacificus gene repertoire was defined as orphan genes without homology in the genomes of other nematode families $[16,37]$. Unbiased genetic screens have identified orphan genes that control important biological processes such as developmental decisions and predatory behavior $[6,42]$. Phylogenomic investigation of ten diplogastrid genomes revealed the evolutionary dynamics of 


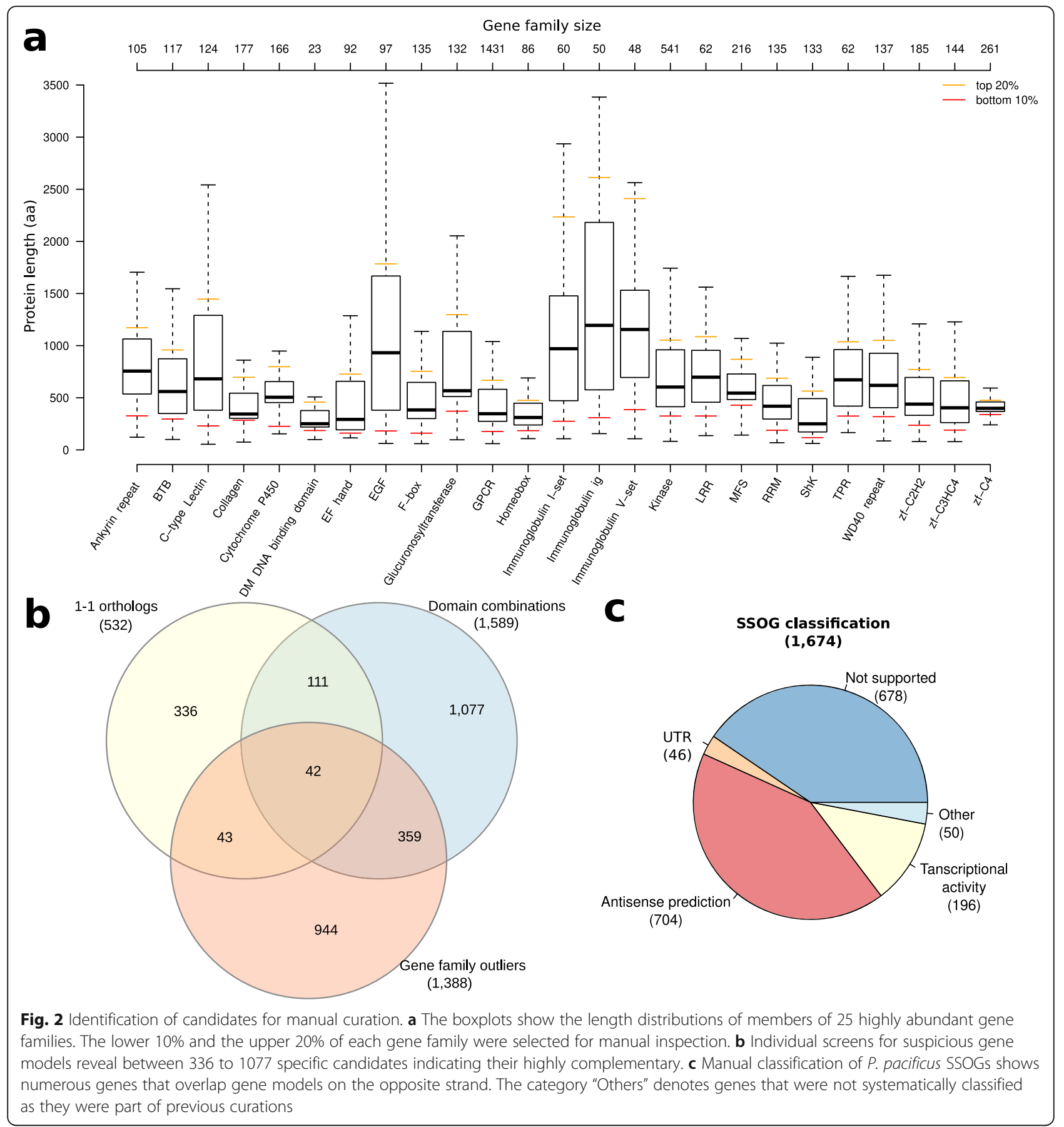

these novel genes and built the framework to dissect the diversity of mechanisms of origin [14, 32]. When we screened for high quality SSOG candidates for origin analysis, we found that the majority of SSOGs had no transcriptomic support. Together with the finding that SSOGs constitute an unusually large age class (phylostratum), this made us wonder to what extent this gene class might possibly be inflated by gene annotation artifacts [14]. Therefore, we revisited 1674 candidates and confirmed that most of them indeed show no evidence of transcription. In addition, we found 704 SSOGs, which overlapped other gene models on the antisense strand and whenever available, strandspecific RNA-seq did not support the SSOG gene model. Even though SSOGs are expected to show little or no evidence of expression and we cannot conclusively argue that these gene models are annotations artifacts (they represent coding potential that might be 


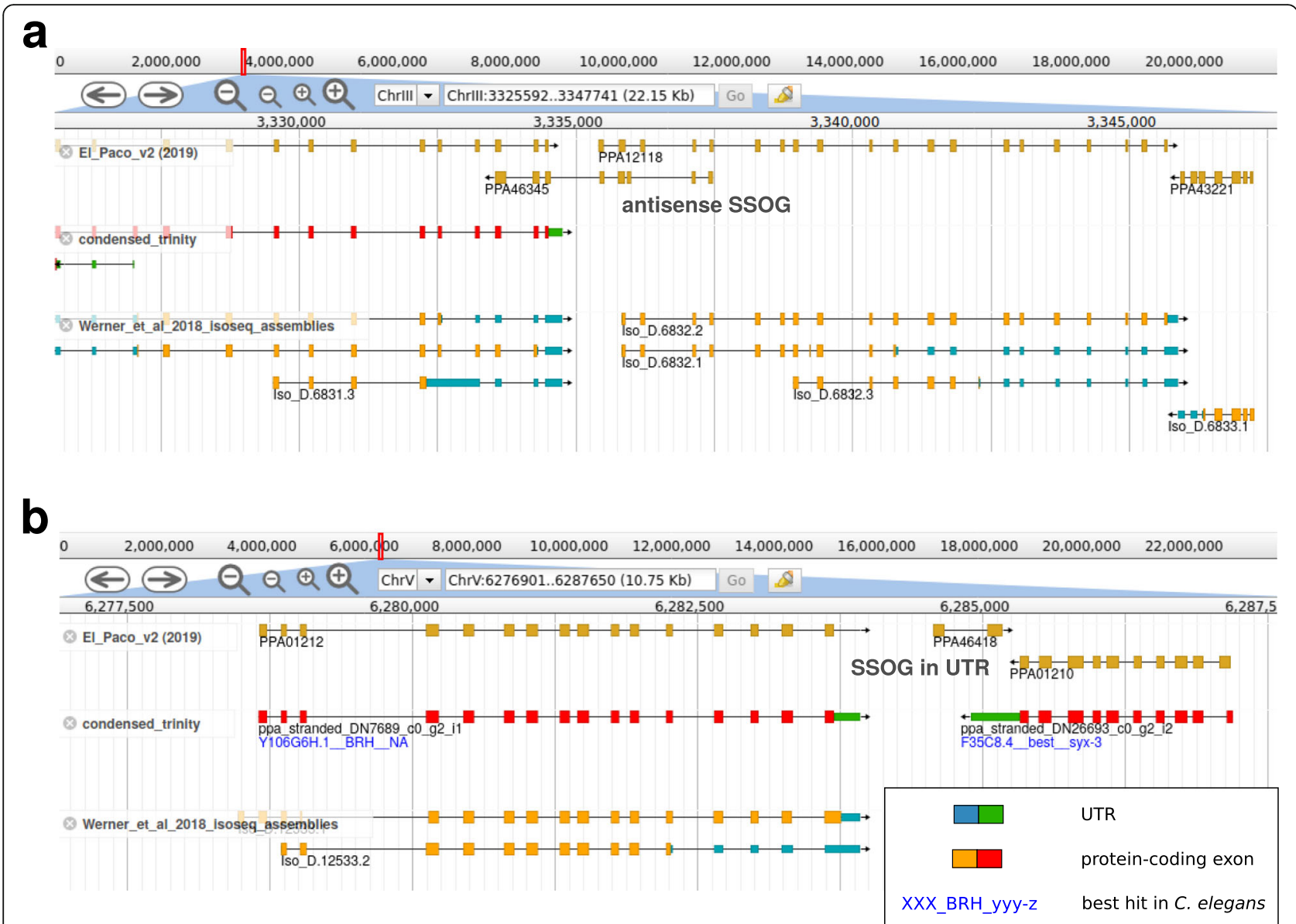

Fig. 3 Examples of unsupported SSOGs. a The P. pacificus SSOG PPA46345 overlaps exons of two other gene models that are well supported by transcriptome assemblies from strand-specific RNA-seq and Iso-seq data. b The P. pacificus SSOG PPA4618 overlaps the UTR of a well supported gene model. The absence of strand-specific transcriptomic support indicates that P. pacificus SSOGs PPA46345 and PPA4618 are likely gene prediction artifacts

used under some conditions), for practical reasons we chose to remove most of the unsupported SSOGs to allow future investigations of orphan origin to start with a set of well supported candidate SSOGs. Thus, we hope that the community-based curation of the $P$. pacificus gene annotations will help future studies in many aspects of evolutionary biology.

\section{Conclusions}

Our work demonstrates that even for non-classical model organisms with small research communities, manual inspection and curation of thousands of genes can be achieved. Thereby numerous comparative genomic screens can be applied to enrich the candidate set for suspicious gene models that actually need to be corrected. The example of the highly curated $P$. pacificus genome emphasizes the effectiveness and scalability of manual curation for many other genome projects including those of nematode animal and plant parasites.

\section{Methods}

Candidate identification based on length comparison of orthologous proteins

We obtained 8348 one-to-one orthologs between $C$. elegans and $P$. pacificus that were predicted based on best reciprocal BLASTP hits in a previous study [23]. We then calculated the protein length ratio between the P. pacificus and C. elegans one-to-one orthologs. In case of multiple isoforms for a given gene, we chose the isoform with the longest protein sequence (WormBase release WS260). Based on an arbitrary cutoff of a twofold difference in protein length between the two species, we identified 532 P. pacificus candidates for manual curation.

\section{Candidate identification based on protein domain content}

We ran the hmmsearch program of the HMMER package (version 3.0, e-value $<0.001$, profiles from PFAM-A.hmm) on protein sets of C. elegans (WS260), P. pacificus (El Paco 
Table 2 Comparison of RNA-seq read alignability

\begin{tabular}{|c|c|c|c|c|}
\hline \multicolumn{2}{|c|}{ P. pacificus RNA-seq samples } & \multicolumn{2}{|c|}{ Successfully assigned alignments (\%) } & \multirow[t]{2}{*}{ Reference } \\
\hline Accession & Description & V2 & V3 & \\
\hline ERR777792 & Mixed-stage on E. coli OP50 & 74.8 & 76.8 & [13] \\
\hline ERR777793 & Mixed-stage on E. coli OP50 & 74.9 & 76.6 & [13] \\
\hline ERR777794 & Mixed-stage on E. coli OP50 & 74.4 & 76.1 & [13] \\
\hline SRR4017216 & Adults on E. coli OP50 & 79.8 & 81.7 & [34] \\
\hline SRR4017217 & Adults on E. coli OP50 & 80.3 & 82.2 & [34] \\
\hline SRR4017218 & Adults on Cryptococcus C3 & 79.6 & 81.6 & [34] \\
\hline SRR4017219 & Adults on Cryptococcus C3 & 79.2 & 81.1 & [34] \\
\hline SRR4017220 & Adults on Cryptococcus C5 & 79.9 & 81.8 & [34] \\
\hline SRR4017221 & Adults on Cryptococcus C5 & 80.7 & 82.6 & {$[34]$} \\
\hline ERR3421261 & Adults on E. coli OP50 & 79.7 & 81.6 & {$[9]$} \\
\hline ERR3421262 & Adults on E. coli OP50 & 79.5 & 81.3 & [9] \\
\hline ERR3421263 & Adults on Novosphingobium L76 & 79.6 & 81.5 & [9] \\
\hline ERR3421264 & Adults on Novosphingobium L76 & 79.5 & 81.5 & [9] \\
\hline SRR2142256 & Adults on E. coli OP50 & 77.8 & 79.8 & {$[35]$} \\
\hline SRR2142257 & Intestines & 72.5 & 74.2 & {$[35]$} \\
\hline
\end{tabular}

The table shows the percentage of assigned reads from 15 RNA-seq experiments for different $P$. pacificus gene annotations

annotation V2), B. xylophilus (WS248), S. ratti (WS260). We counted occurrences of protein domains and defined as candidates, domain combinations that are unique to $P$. pacificus and occur at low frequencies (less than ten times). This yielded 1589 candidates with atypical protein domain combinations. Next, we selected 25 highly abundant gene families such as collagens and C-type lectins that were defined by a PFAM domain and classified further candidate proteins if their length fell under the first or above the eighth decile of the length distribution of all members of a given gene family. This identified 1388 candidate genes for manual curation.

\section{Identification and curation of $P$. pacificus species-specific orphan genes}

We defined $P$. pacificus SSOGs by BLASTP searches of the $P$. pacificus proteins (version: El Paco annotation V2) against annotated protein sets and predicted open reading frames (ORFs) in assembled transcripts of $P$. exspectatus, $P$. arcanus, $P$. maxplancki, and $P$. japonica [26, 32]. This identified 1988 (7\%) P. pacificus SSOGs without a BLASTP hit in any of the reference data sets (e-value <0.001). Three hundred fourteen SSOGs showed transcriptomic support as they had a BLASTP hit in ORFs of the $P$. pacificus transcriptome assembly. The remaining 1674 were defined as SSOGs without transcriptomic support and were thus considered as candidates for manual curation.

\section{Community-based manual curation of gene models}

Community-based gene curation was performed as described in our pilot study [23]. In short, candidate lists were shared in online spreadsheets and individual genes were visually inspected in the jbrowse genome browser instance on http://www.pristionchus.org [44]. Based on available transcriptomic resources, which include RNA-seq data from different developmental stages, strand-specific transcriptome assemblies from mixed-stage cultures [25, 26], and iso-seq data [27], community curators were trained to evaluate whether a locus was well covered by transcriptomic data and in case of evidence for an artificial gene fusion to propose the replacement of the original gene model by assembled transcripts. If the genomic neighborhood of the candidate genes showed obvious inconsistencies between original gene models and transcriptome data, we eventually curated such neighboring genes. However, we omitted any gene that was curated in our previous study, as these changes were not yet fully implemented in the latest WormBase release WS177 of $P$. pacificus and we wanted to avoid version conflicts. While for most candidate genes, we did not propose any correction in case that available transcriptomic data was insufficient to make a conclusive statement, in the case of SSOGs, we typically removed the gene model if not at least some RNA-seq data supported the gene structure.

\section{Quality assessment of gene annotations}

In order to evaluate the quality of gene annotations, we ran the BUSCO program (version 3.0.1) in protein mode 
(option: -m prot) against the nematode_odb9 data set ( $N=982$ orthologs) [24]. To test whether the new set of gene annotations better captures RNA-seq data, we downloaded 15 RNA-seq data sets from the European Nucleotide Archive and aligned these data sets against the $P$. pacificus reference genome (version: El Paco) with the help of the STAR aligner (version: 2.5.4b, default options, reference was the $P$. pacificus genome without any gene annotation) [45]. Next, we quantified the percentage of alignments that could be assigned to gene annotations using the featureCounts function of the Rsubread library in $\mathrm{R}$ (version 4.0.0).

\section{Supplementary information}

Supplementary information accompanies this paper at https://doi.org/10. 1186/s12864-020-07100-0.

Additional file 1: Figure S1. Candidate genes for incorrect gene annotations in C. elegans.

\section{Abbreviations}

BUSCO: Benchmarking Universal Single Copy Orthologs; ORF: Open Reading Frame; SSOG: Species-specific Orphan Gene; UTR : Untranslated Region

\section{Acknowledgements}

The authors would like to thank the complete Pristionchus community for their long-term interest in studying P. pacificus and thus motivating this work.

\section{Authors' contributions}

Conceptualization, C.R.; Investigation, C.R., M.A., H.W., C.W., T.L. and G.E.; Data curation, C.R., M.A., H.W., C.W., T.L. and G.E.; Visualization, C. R.; Writing original draft, C.R.; Writing - review \& editing, C.R., and R.J.S.; Project administration, C. R.; Supervision, C.R. and R.J.S.; Funding acquisition, R.J.S. The author(s) read and approved the final manuscript.

\section{Funding}

This work was funded by the Max Planck Society. Open Access funding enabled and organized by Projekt DEAL.

\section{Availability of data and materials}

The new set of $P$. pacificus gene annotations (version: El Paco gene annotation V3) was submitted as an update of the existing whole-genome shotgun project at ENA/Genbank: ABKE00000000. The annotations were also submitted to WormBase where they will be published following further curation and they are publicly available at http://www.pristionchus.org/ download/.

\section{Ethics approval and consent to participate}

Not applicable.

\section{Consent for publication}

Not applicable.

\section{Competing interests}

The authors declare that they have no competing interests.

Received: 3 August 2020 Accepted: 23 September 2020

Published online: 12 October 2020

\section{References}

1. Sommer RJ, Carta L, Kim S-Y, Sternberg PW. Morphological, genetic and molecular description of Pristionchus pacificus sp. n.(Nematoda: Neodiplogasteridae). Fundam Appl Nematol. 1996;19:511-22.

2. Sommer RJ. The future of evo-devo: model systems and evolutionary theory. Nat Rev Genet. 2009;10:416-22. https://doi.org/10.1038/nrg2567.
3. Kieninger MR, Ivers NA, Rödelsperger C, Markov GV, Sommer RJ, Ragsdale EJ. The nuclear hormone receptor NHR-40 acts downstream of the sulfatase EUD-1 as part of a developmental plasticity switch in Pristionchus. Curr Biol. 2016:26:2174-9.

4. Sieriebriennikov B, Prabh N, Dardiry M, Witte $H$, Röseler W, Kieninger MR, et al. A developmental switch generating phenotypic plasticity is part of a conserved multi-gene locus. Cell Rep. 2018;23:2835-43.e4.

5. Sieriebriennikov B, Sun S, Lightfoot JW, Witte H, Moreno E, et al. Conserved nuclear hormone receptors controlling a novel plastic trait target fastevolving genes expressed in a single cell. PLoS Genet. 2020;16:e1008687. https://doi.org/10.1371/journal.pgen.1008687.

6. Lightfoot JW, Wilecki M, Rödelsperger C, Moreno E, Susoy V, Witte H, et al. Small peptide-mediated self-recognition prevents cannibalism in predatory nematodes. Science. 2019;364:86-9. https://doi.org/10.1126/science.aav9856.

7. Moreno E, McGaughran A, Rödelsperger C, Zimmer M, Sommer RJ. Oxygeninduced social behaviours in Pristionchus pacificus have a distinct evolutionary history and genetic regulation from Caenorhabditis elegans. Proc Biol Sci. 2016;283:20152263.

8. Moreno E, Sieriebriennikov B, Witte H, Rödelsperger C, Lightfoot JW, Sommer RJ. Regulation of hyperoxia-induced social behaviour in Pristionchus pacificus nematodes requires a novel cilia-mediated environmental input. Sci Rep. 2017;7:17550.

9. Akduman N, Lightfoot JW, Röseler W, Witte H, Lo W-S, et al. Bacterial vitamin B12 production enhances nematode predatory behavior. ISME J. 2020;14:1494-507. https://doi.org/10.1038/s41396-020-0626-2.

10. Meyer JM, Baskaran P, Quast C, Susoy V, Rödelsperger C, Glöckner FO, et al. Succession and dynamics of Pristionchus nematodes and their microbiome during decomposition of Oryctes borbonicus on La Réunion Island. Environ Microbiol. 2017;19:1476-89. https://doi.org/10.1111/1462-2920.13697.

11. Akduman N, Rödelsperger C, Sommer RJ. Culture-based analysis of Pristionchus-associated microbiota from beetles and figs for studying nematode-bacterial interactions. PLoS One. 2018;13:e0198018.

12. Weller AM, Rödelsperger C, Eberhardt G, Molnar RI, Sommer RJ. Opposing forces of a/T-biased mutations and G/C-biased gene conversions shape the genome of the nematode Pristionchus pacificus. Genetics. 2014;196:1145-52. https://doi.org/10.1534/genetics.113.159863.

13. Baskaran P, Rödelsperger C. Microevolution of duplications and deletions and their impact on gene expression in the nematode Pristionchus pacificus. PLoS One. 2015;10:e0131136.

14. Prabh N, Rödelsperger C. De novo, divergence, and mixed origin contribute to the emergence of orphan genes in Pristionchus nematodes. G3: Genes|Genomes| Genetics. 2019;9:2277-86. https://doi.org/10.1534/g3.119.400326..

15. Dieterich C, Clifton SW, Schuster LN, Chinwalla A, Delehaunty K, Dinkelacker I, et al. The Pristionchus pacificus genome provides a unique perspective on nematode lifestyle and parasitism. Nat Genet. 2008;40:1193-8.

16. Borchert N, Dieterich C, Krug K, Schütz W, Jung S, Nordheim A, et al. Proteogenomics of Pristionchus pacificus reveals distinct proteome structure of nematode models. Genome Res. 2010;20:837-46.

17. Rödelsperger C, Meyer JM, Prabh N, Lanz C, Bemm F, Sommer RJ. Singlemolecule sequencing reveals the chromosome-scale genomic architecture of the nematode model organism Pristionchus pacificus. Cell Rep. 2017;21: 834-44.

18. Cantarel BL, Korf I, Robb SMC, Parra G, Ross E, Moore B, et al. MAKER: an easy-to-use annotation pipeline designed for emerging model organism genomes. Genome Res. 2008;18:188-96.

19. Korf I. Gene finding in novel genomes. BMC Bioinformatics. 2004;5:59.

20. Stanke M, Keller O, Gunduz I, Hayes A, Waack S, Morgenstern B. AUGUSTUS: ab initio prediction of alternative transcripts. Nucleic Acids Res. 2006;34(Web Server issue):W435-9.

21. Markov GV, Meyer JM, Panda O, Artyukhin AB, Claaßen M, Witte $H$, et al. Functional conservation and divergence of daf-22 paralogs in Pristionchus pacificus dauer development. Mol Biol Evol. 2016;33:2506-14.

22. Okumura M, Wilecki M, Sommer RJ. Serotonin drives predatory feeding behavior via synchronous feeding rhythms in the nematode. G3. 2017;7: 3745-55.

23. Rödelsperger $C$, Athanasouli $M$, Lenuzzi $M$, Theska $T$, Sun $S$, Dardiry $M$, et al. Crowdsourcing and the feasibility of manual gene annotation: a pilot study in the nematode Pristionchus pacificus. Sci Rep. 2019:9:18789.

24. Simão FA, Waterhouse RM, loannidis P, Kriventseva EV, Zdobnov EM. BUSCO: assessing genome assembly and annotation completeness with single-copy orthologs. Bioinformatics. 2015;31:3210-2. 
25. Rödelsperger C, Menden K, Serobyan V, Witte H, Baskaran P. First insights into the nature and evolution of antisense transcription in nematodes. BMC Evol Biol. 2016;16:165.

26. Rödelsperger C, Röseler W, Prabh N, Yoshida K, Weiler C, Herrmann M, et al, Phylotranscriptomics of Pristionchus nematodes reveals parallel gene loss in six hermaphroditic lineages. Curr Biol. 2018;28:3123-7.e5.

27. Werner MS, Sieriebriennikov B, Prabh N, Loschko T, Lanz C, Sommer RJ. Young genes have distinct gene structure, epigenetic profiles, and transcriptional regulation. Genome Res. 2018;28:1675-87.

28. Markov GV, Baskaran P, Sommer RJ. The same or not the same: lineagespecific gene expansions and homology relationships in multigene families in nematodes. J Mol Evol. 2015;80:18-36.

29. Rödelsperger C, Prabh N, Sommer RJ. New gene origin and deep taxon phylogenomics: opportunities and challenges. Trends Genet. 2019;35: 914-22.

30. Kikuchi T, Cotton JA, Dalzell JJ, Hasegawa K, Kanzaki N, McVeigh P, et al. Genomic insights into the origin of parasitism in the emerging plant pathogen Bursaphelenchus xylophilus. PLoS Pathog. 2011;7:e1002219.

31. Hunt VL, Tsai IJ, Coghlan A, Reid AJ, Holroyd N, Foth BJ, et al. The genomic basis of parasitism in the Strongyloides clade of nematodes. Nat Genet. 2016;48:299-307.

32. Prabh N, Roeseler W, Witte H, Eberhardt G, Sommer RJ, Rödelsperger C. Deep taxon sampling reveals the evolutionary dynamics of novel gene families in Pristionchus nematodes. Genome Res. 2018;28:1664-74.

33. Baskaran P, Rödelsperger C, Prabh N, Serobyan V, Markov GV, Hirsekorn A, et al. Ancient gene duplications have shaped developmental stage-specific expression in Pristionchus pacificus. BMC Evol Biol. 2015;15:185.

34. Sanghvi GV, Baskaran P, Röseler W, Sieriebriennikov B, Rödelsperger C, Sommer RJ. Life history responses and gene expression profiles of the nematode Pristionchus pacificus cultured on Cryptococcus yeasts. PLoS One. 2016;11:e0164881. https://doi.org/10.1371/journal.pone.0164881.

35. Lightfoot JW, Chauhan VM, Aylott JW, Rödelsperger C. Comparative transcriptomics of the nematode gut identifies global shifts in feeding mode and pathogen susceptibility. BMC Res Notes. 2016;9:142.

36. Holt C, Yandell M. MAKER2: an annotation pipeline and genome-database management tool for second-generation genome projects. BMC Bioinformatics. 2011:12:491.

37. Rödelsperger C, Sommer RJ. Computational archaeology of the Pristionchus pacificus genome reveals evidence of horizontal gene transfers from insects. BMC Evol Biol. 2011;11. https://doi.org/10.1186/1471-2148-11-239.

38. Rae R, Witte H, Rödelsperger C, Sommer RJ. The importance of being regular: Caenorhabditis elegans and Pristionchus pacificus defecation mutants are hypersusceptible to bacterial pathogens. Int J Parasitol. 2012:42:747-53. https://doi.org/10.1016/j.ijpara.2012.05.005.

39. Bumbarger DJ, Riebesell M, Rödelsperger C, Sommer RJ. System-wide rewiring underlies behavioral differences in predatory and bacterial-feeding nematodes. Cell. 2013;152:109-19.

40. Hong RL, Riebesell M, Bumbarger DJ, Cook SJ, Carstensen HR, Sarpolaki T, et al. Evolution of neuronal anatomy and circuitry in two highly divergent nematode species. Elife. 2019;8. https://doi.org/10.7554/eLife.47155.

41. Rödelsperger C. Comparative genomics of gene loss and gain in Caenorhabditis and other nematodes. Comparative Genomics. 2018:419-32. https://doi.org/10.1007/978-1-4939-7463-4_16.

42. Mayer MG, Rödelsperger C, Witte H, Riebesell M, Sommer RJ. The orphan gene dauerless regulates dauer development and intraspecific competition in nematodes by copy number variation. PLoS Genet. 2015;11:e1005146.

43. Prabh N, Rödelsperger C. Are orphan genes protein-coding, prediction artifacts, or non-coding RNAs? BMC Bioinformatics. 2016;17:226.

44. Skinner ME, Uzilov AV, Stein LD, Mungall CJ, Holmes IH. JBrowse: a nextgeneration genome browser. Genome Res. 2009;19:1630-8. https:/doi.org/ 10.1101/gr.094607.109.

45. Dobin A, Davis CA, Schlesinger F, Drenkow J, Zaleski C, Jha S, et al. STAR: ultrafast universal RNA-seq aligner. Bioinformatics. 2013;29:15-21.

\section{Publisher's Note}

Springer Nature remains neutral with regard to jurisdictional claims in published maps and institutional affiliations.

\section{Ready to submit your research? Choose BMC and benefit from:}

- fast, convenient online submission

- thorough peer review by experienced researchers in your field

- rapid publication on acceptance

- support for research data, including large and complex data types

- gold Open Access which fosters wider collaboration and increased citations

- maximum visibility for your research: over $100 \mathrm{M}$ website views per year

At BMC, research is always in progress.

Learn more biomedcentral.com/submissions 